\title{
Fractura del poste tibial en la artroplastia total de rodilla estabilizada a posterior. Presentación de dos casos
}

\author{
César Pesciallo, Fernando H. Lopreite, Germán Garabano, Fernando Tillet, Joaquín Rodríguez, Hernán del Sel \\ Servicio de Ortopedia y Traumatología, Hospital Británico de Buenos Aires, Ciudad Autónoma de Buenos Aires, Argentina
}

\begin{abstract}
RESUMEN
La fractura del poste tibial luego de un reemplazo total de rodilla es una complicación poco frecuente e incapacitante. Presentamos a dos pacientes con fractura del poste tibial no traumática, que acudieron con dolor más inestabilidad en recurvatum, de forma repentina, después de reemplazos articulares con buen funcionamiento previo. El primer paciente se presentó a los 12 años del reemplazo total de rodilla primario y el segundo, a los 14 meses. Ambos tenían el mismo modelo de prótesis estabilizada a posterior. Los dos pacientes fueron sometidos a una revisión con prótesis abisagrada rotatoria (Endo-Model NR®, Waldemar Link, EE.UU.), sin recidiva de la deformidad.
\end{abstract}

Palabras clave: Rotura; poste tibial; recurvatum; reemplazo total de rodilla; prótesis estabilizada a posterior.

Nivel de Evidencia: IV

\section{Fracture of a Polyethylene Tibial Post in Posterior Stabilized Total Knee Replacement. Two Case Reports}

\section{ABSTRACT}

Tibial post fracture after posterior stabilized total knee replacement (TKR) is a uncommon and disabling complication. We report two cases of non-traumatic tibial post fracture, which presented with sudden pain and recurvatum instability in joint replacements with good previous function. For the first case, the presentation time was 12 years from the primary TKR, and for the second it was 14 months. Both presented the same model of a posterior stabilized prosthesis. In both cases, a revision TKR was performed with a rotating hinge prosthesis (Endo-Model NR囚, Waldemar Link), without recurrence of the deformity.

Key words: Tibial post fracture; recurvatum; total knee replacement; posterior stabilized total knee replacement.

Level of Evidence: IV

\section{INTRODUCCIÓN}

Múltiples etiologías contribuyen a las fallas de un reemplazo total de rodilla y la inestabilidad es la causa de revisión en el 10-22\% de los casos. ${ }^{1-3}$ La inestabilidad se puede clasificar de la siguiente manera: en flexión, en extensión y genu recurvatum. ${ }^{4}$ La aparición de genu recurvatum después de un reemplazo total de rodilla es poco frecuente. , $^{1,2,4}$

Presentamos a dos pacientes con rotura del poste tibial e inestabilidad en recurvatum.

\section{CASO CLÍNICO 1}

Hombre de 77 años, con antecedentes clínicos de obesidad, diabetes, insuficiencia renal crónica, hipertensión arterial, insuficiencia cardíaca, pie de Charcot bilateral y marcapasos por un bloqueo de la conducción cardíaca. Había sido sometido a un reemplazo total de rodilla izquierda por genu varo artrósico, en noviembre de 2006 . En esa oportunidad, se le colocó una prótesis estabilizada a posterior (Scorpio®, Stryker, NJ, EE.UU.) y tuvo una buena evolución durante casi 12 años (Figuras 1 y 2). 


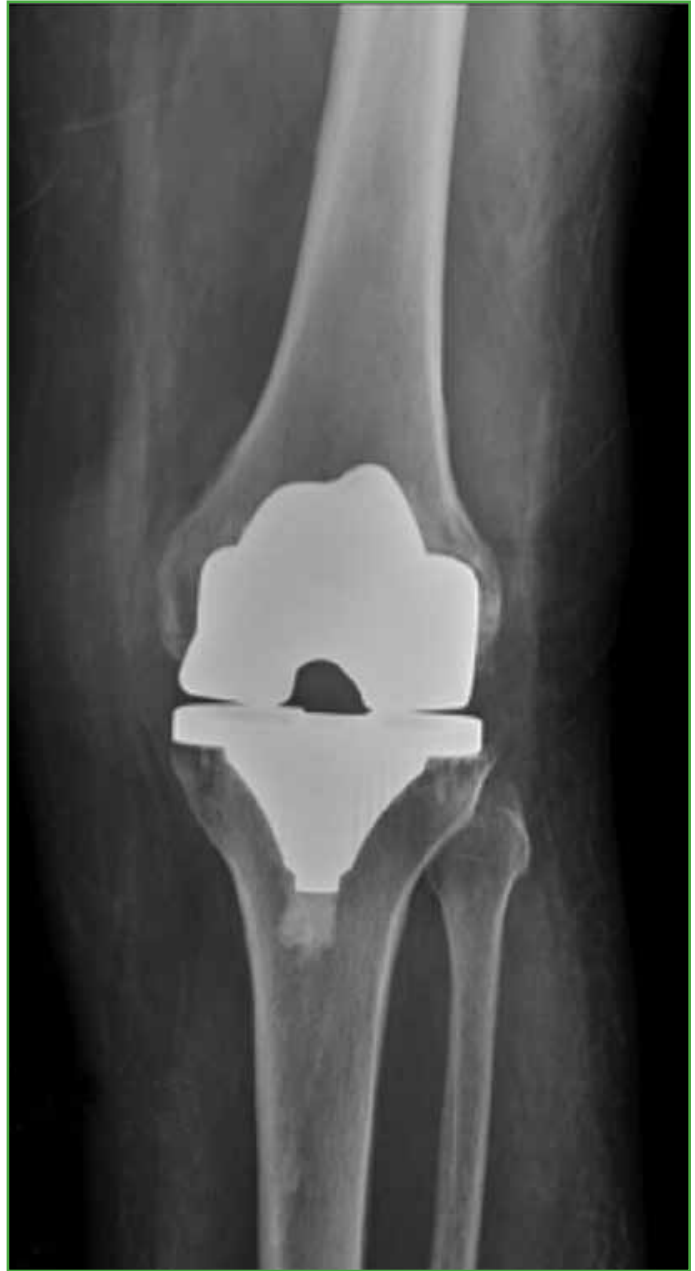

Figura 1. Radiografía de frente a los 11 años del reemplazo total de rodilla. Eje correcto y componentes de la prótesis en posición adecuada, no hay signos de demarcación o aflojamiento.

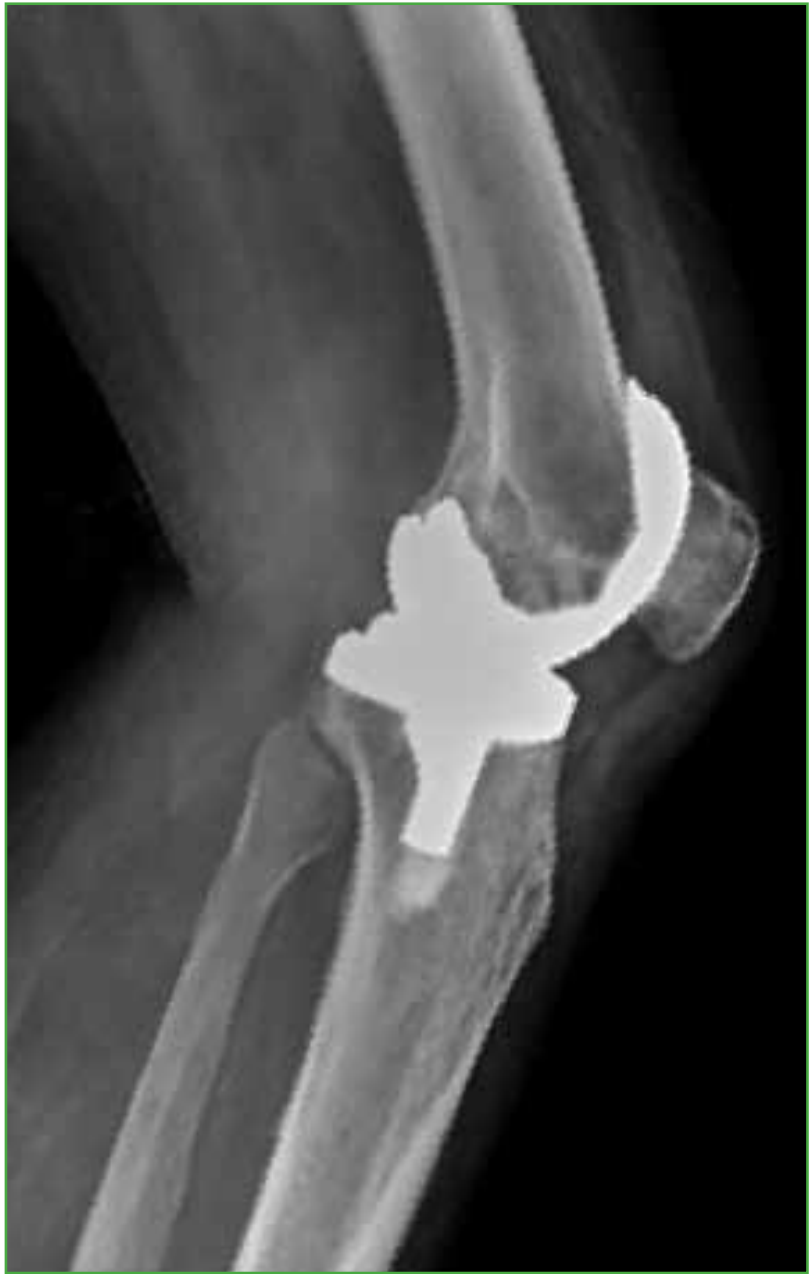

Figura 2. Radiografía de perfil a los 11 años de la cirugía. Correcto posicionamiento de los componentes, sin recurvatum.

En octubre de 2018, consulta por dolor e inestabilidad de rodilla en recurvatum que le imposibilita la marcha. En el examen físico, se observó una rodilla estable al varo-valgo, con movilidad pasiva de $0-100^{\circ}$ y movilidad activa de $10-100^{\circ}$, y una clara debilidad en la fuerza muscular del cuádriceps.

En las radiografías de frente, se visualizó un implante en posición y alineación correctas, sin signos de demarcación. En las imágenes de perfil, se pudo comprobar el recurvatum de aproximadamente $20^{\circ}$ (Figuras 3 y 4 ). 


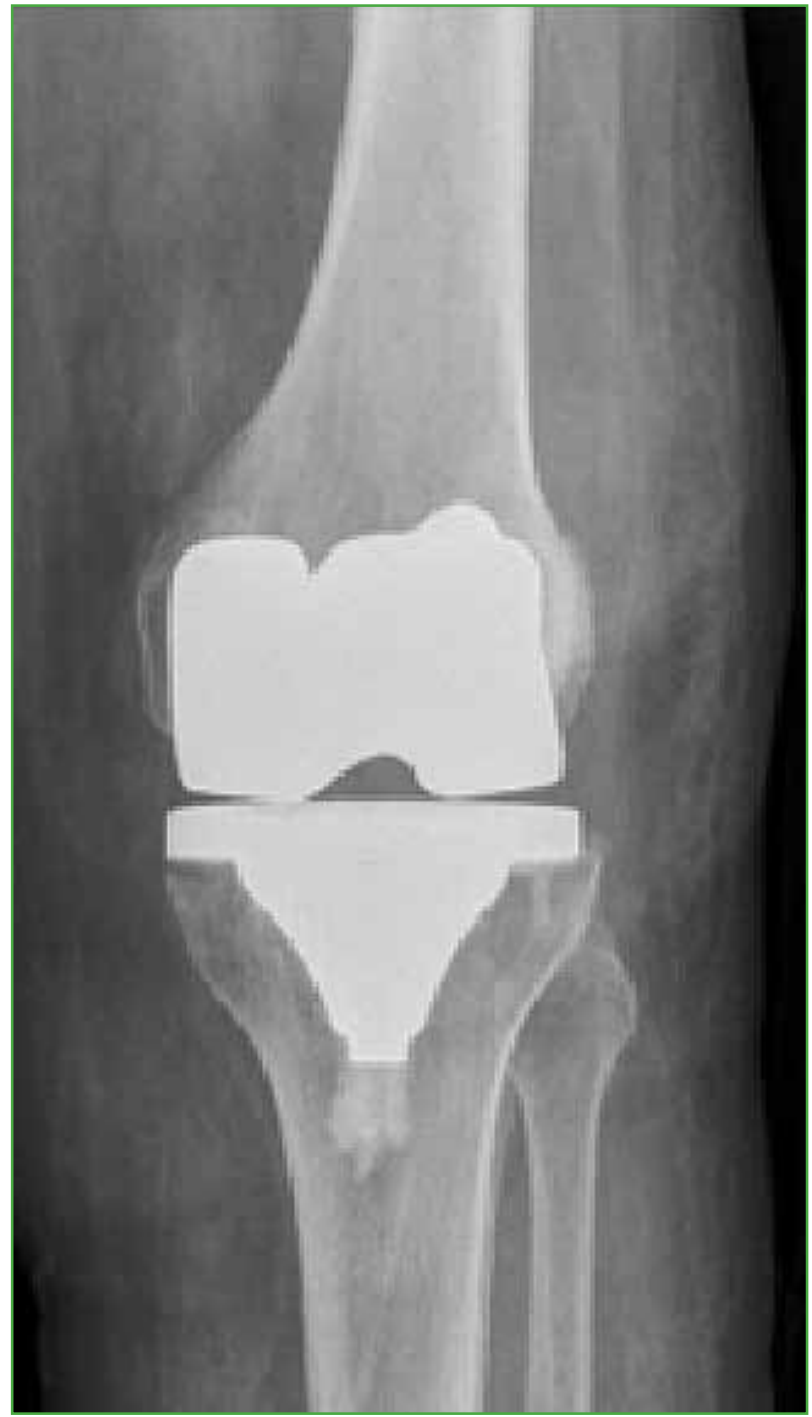

Figura 3. Radiografía de frente del reemplazo total de rodilla. Eje correcto, sin signos de aflojamiento.

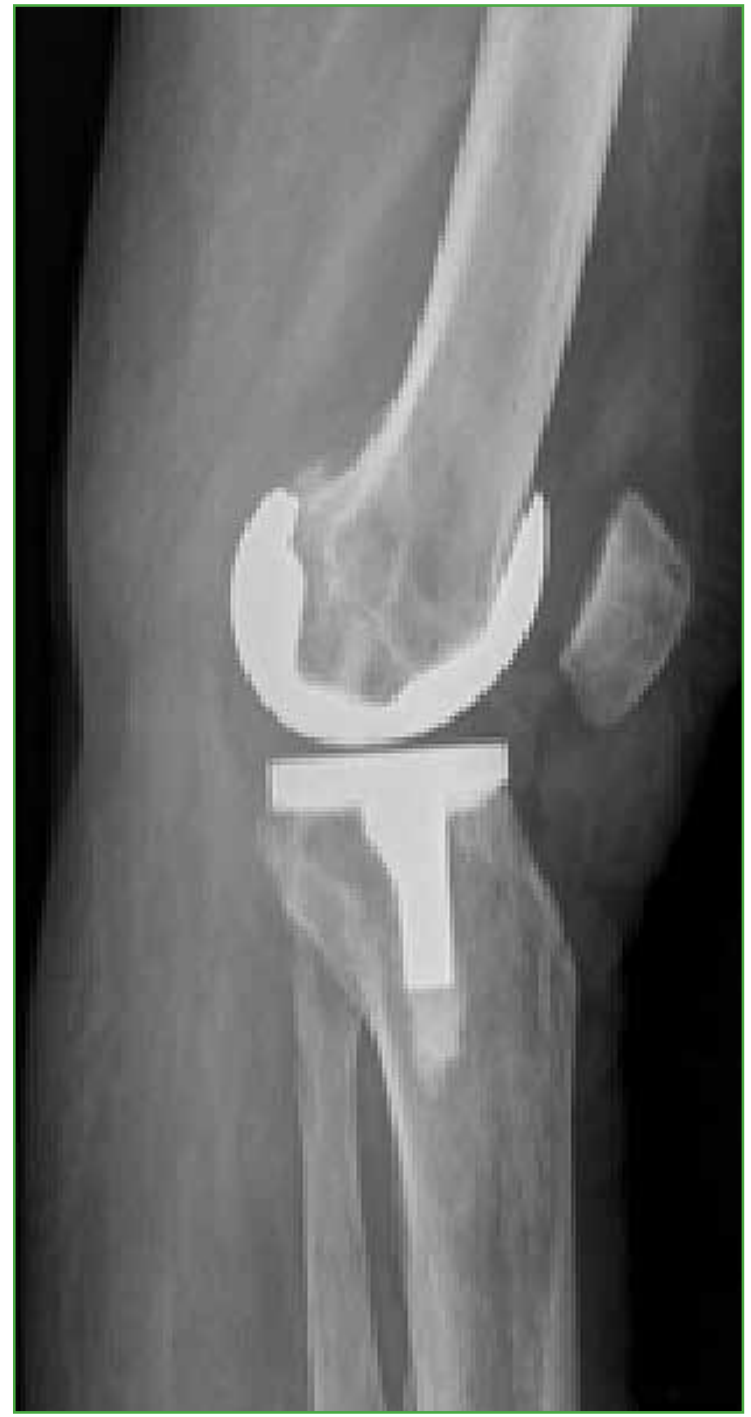

Figura 4. Radiografía de perfil. Correcto posicionamiento de los componentes, con recurvatum.

Durante la cirugía, se detectó la rotura del poste central del inserto de polietileno (liner) (Figura 5). Se procedió a retirar la prótesis primaria para luego implantar una prótesis abisagrada con plataforma rotatoria (Endo-Model NR®, Waldemar Link GmbH \& Co. KG, Hamburgo, Alemania), con buena evolución hasta la fecha (15 meses después de la cirugía) (Figuras 6 y 7 ). 


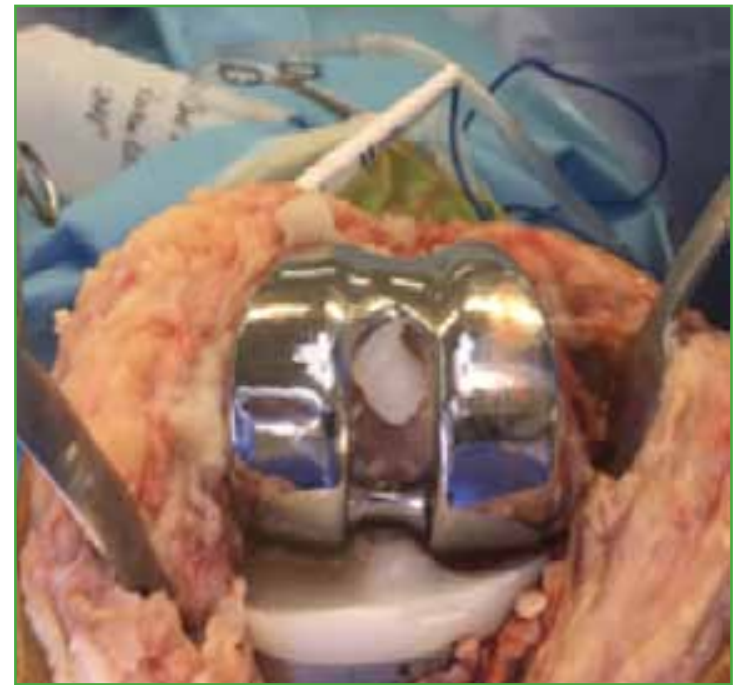

Figura 5. Imagen intraoperatoria. Se observa la rotura del poste tibial.

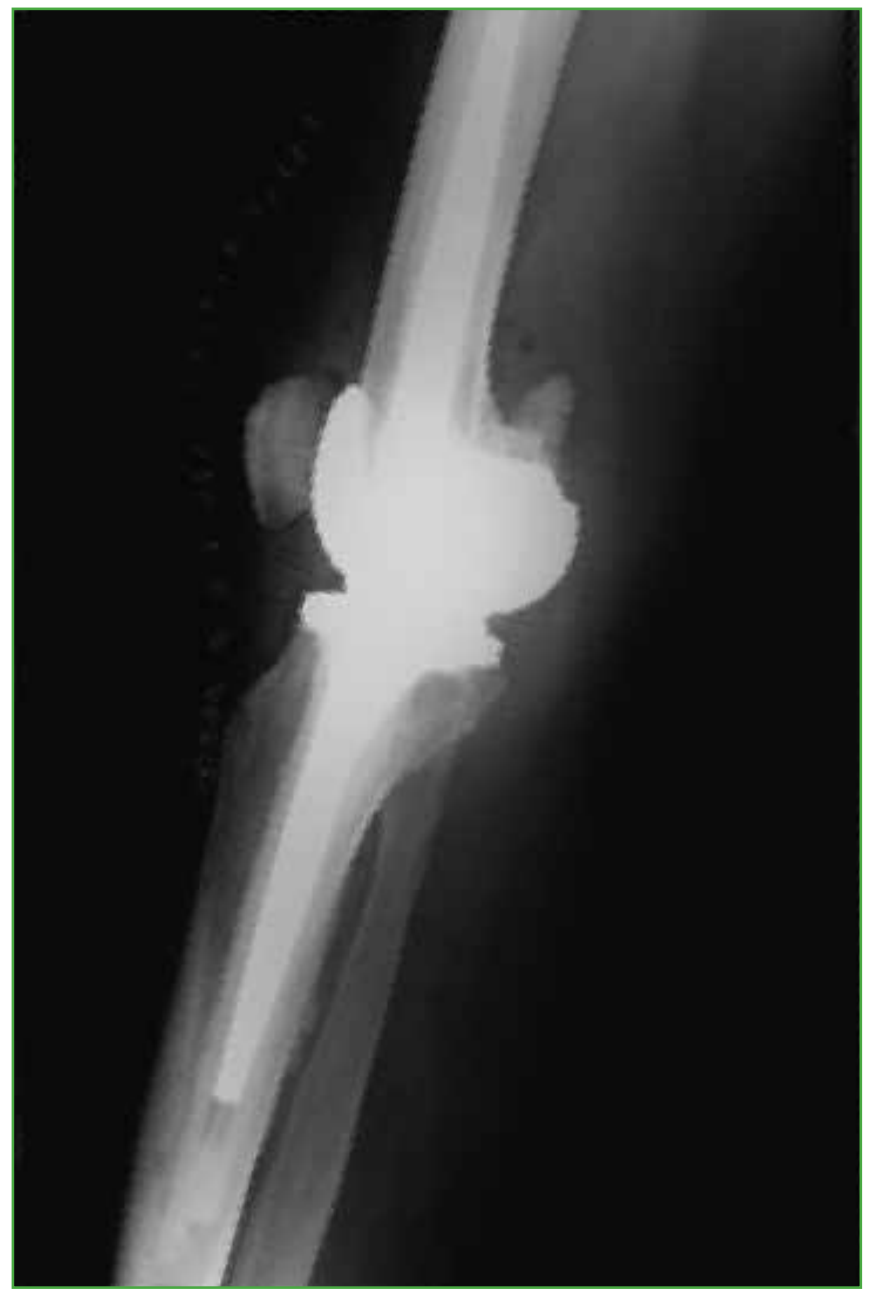

Figura 6. Radiografía de perfil. Control de la revisión con una prótesis abisagrada rotatoria.

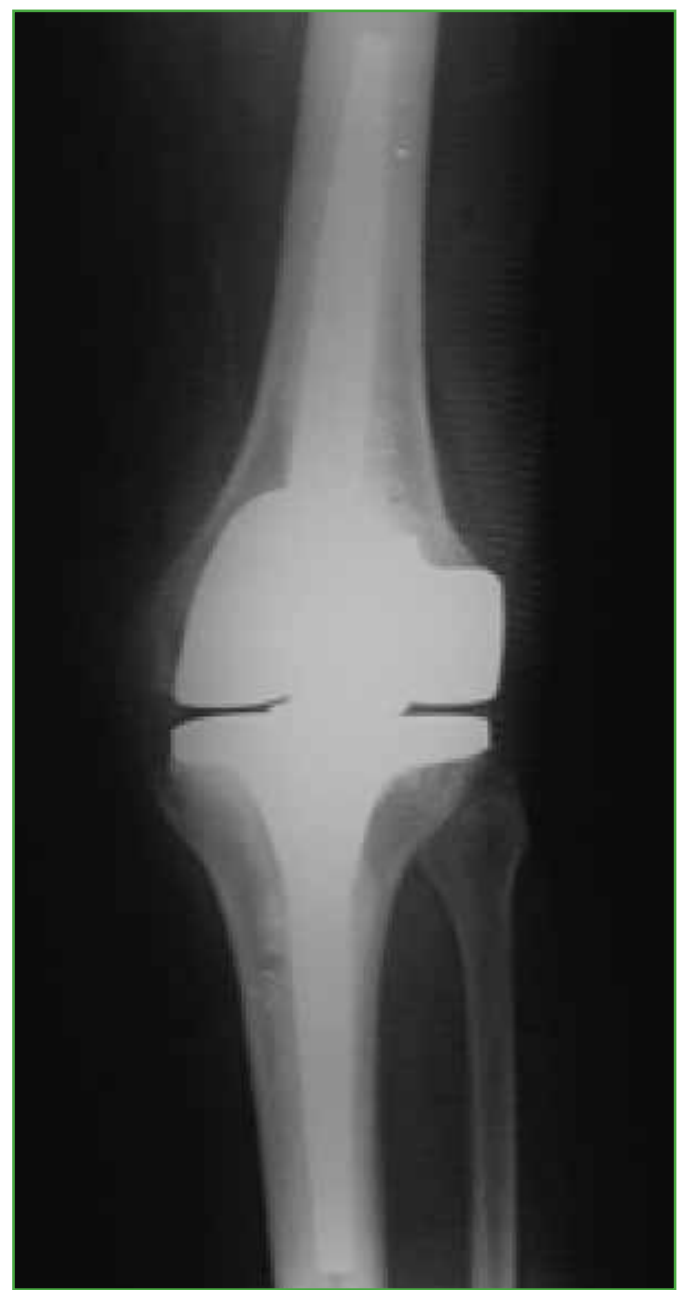

Figura 7. Radiografía de frente de la revisión. Eje adecuado. 


\section{CASO CLÍNICO 2}

Mujer de 64 años, con antecedentes de artritis reumatoide de 30 años de evolución. En abril de 2014, fue sometida a un reemplazo total de rodilla derecha, en otro centro. Se le implantó una prótesis Scorpio® (Stryker®, NJ, NY, EE.UU.).

La paciente tenía una rodilla con buena función clínica, caminaba sin asistencia y sin limitación desde el tercer mes posterior a la cirugía y, en las radiografías de control, se observó un eje correcto sin demarcación de la prótesis.

Según los datos obtenidos, tenía una movilidad pasiva de $0-100^{\circ}$ y una movilidad activa de $10-100^{\circ}$.

Concurrió a nuestro centro en marzo de 2016 (aproximadamente 2 años después de la intervención) por inestabilidad de la prótesis en recurvatum que le impedía la marcha; no refirió un antecedente traumático (Figuras 8 y 9).

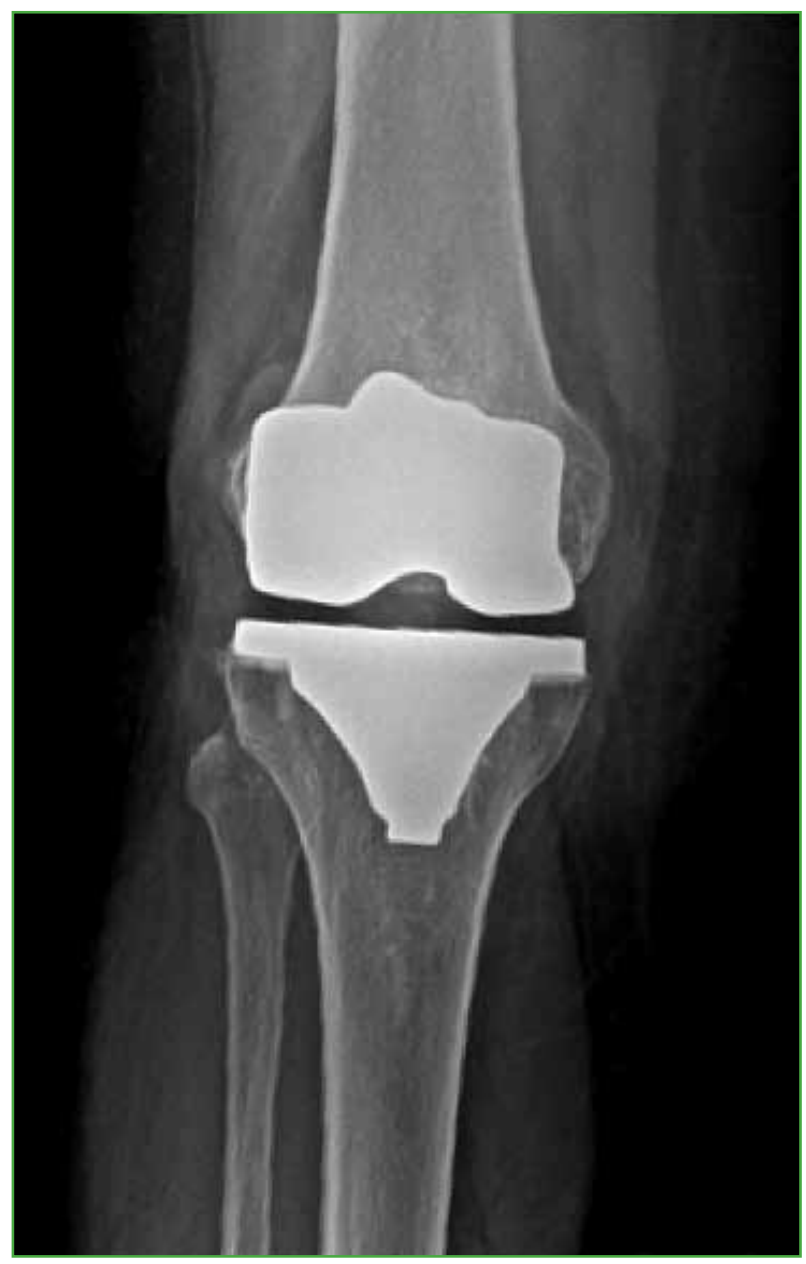

Figura 8. Radiografía de frente del reemplazo total de rodilla. Eje correcto, componentes protésicos en posición correcta, sin signos de demarcación o aflojamiento.

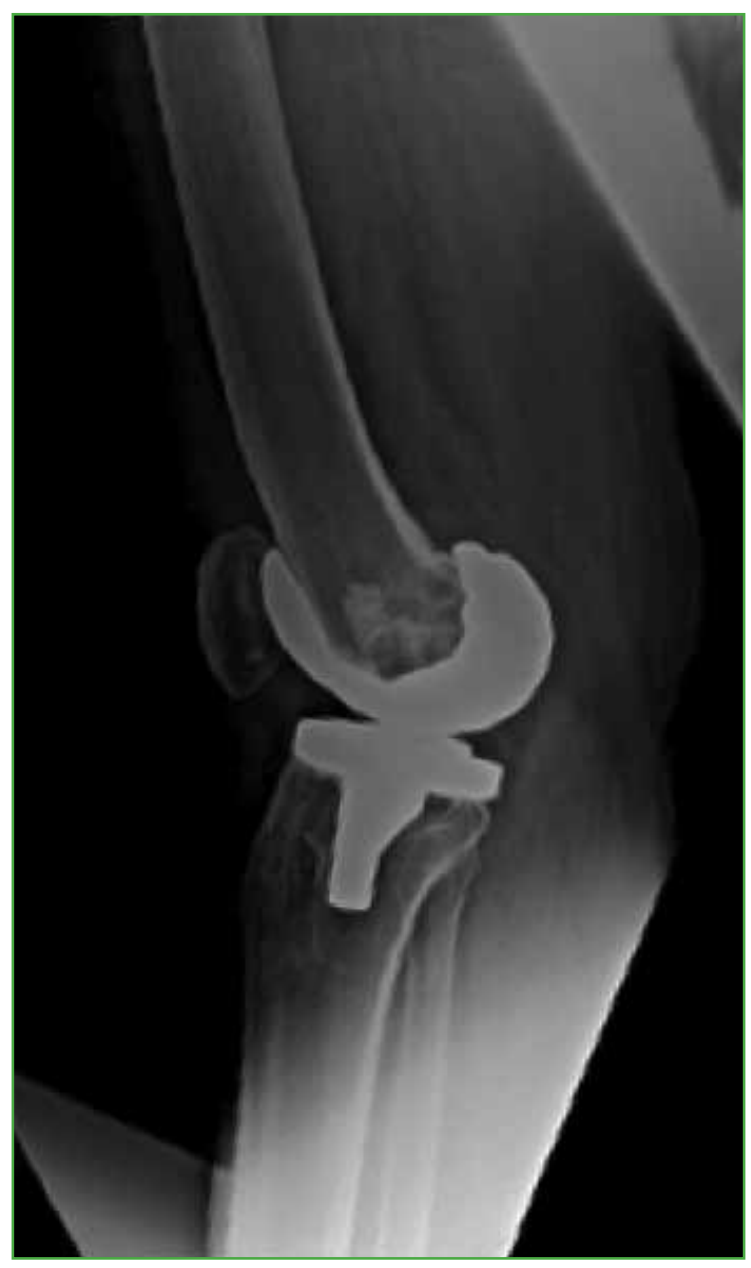

Figura 9. Radiografía de perfil. Correcto posicionamiento de los componentes, con recurvatum. 
En abril de 2016, se procedió a la revisión de la prótesis y se colocó una prótesis abisagrada rotatoria EndoModel@. La paciente ha tenido una buena evolución hasta la fecha (34 meses poscirugía) (Figuras 10 y 11).

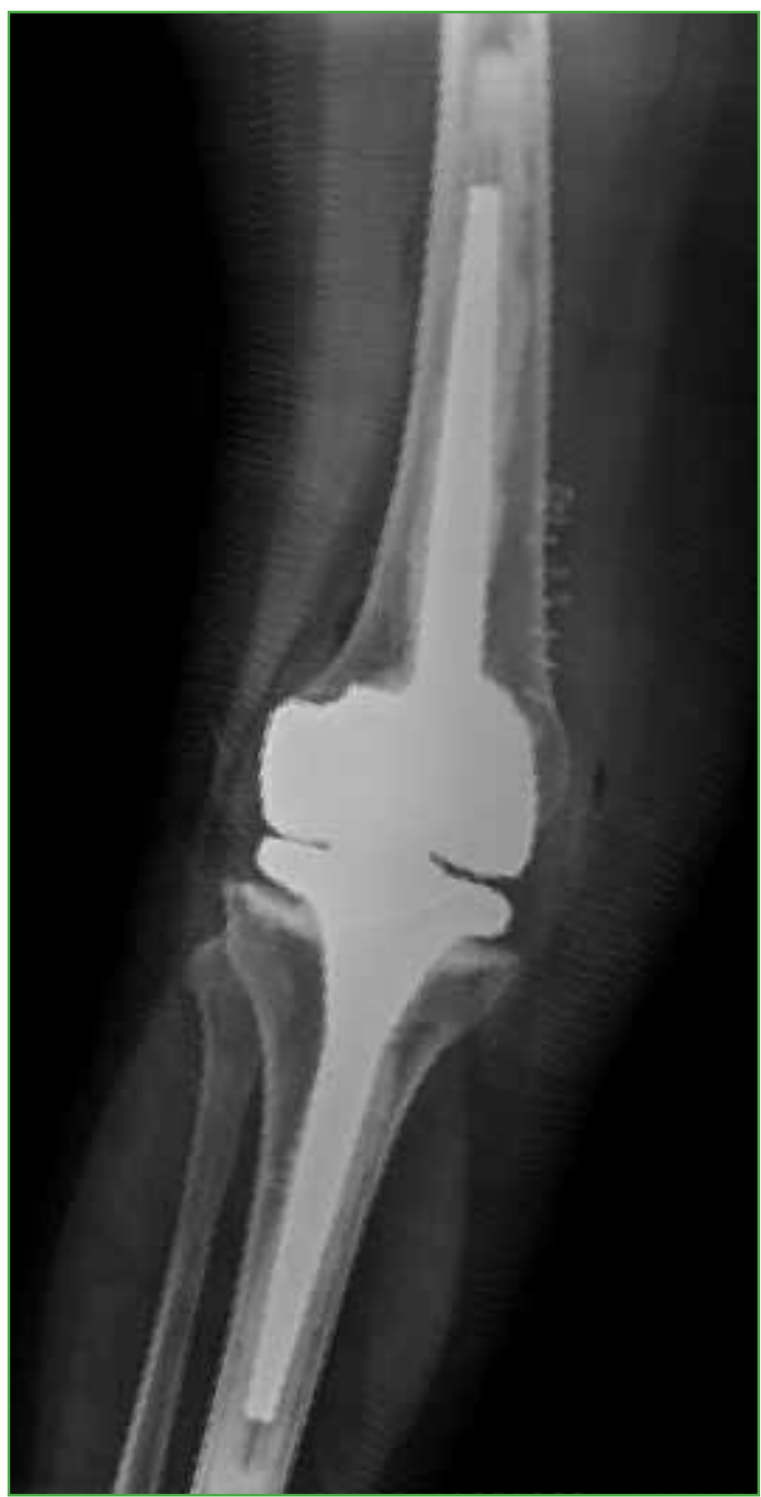

Figura 10. Radiografía de frente. Control de la revisión con una prótesis abisagrada rotatoria.

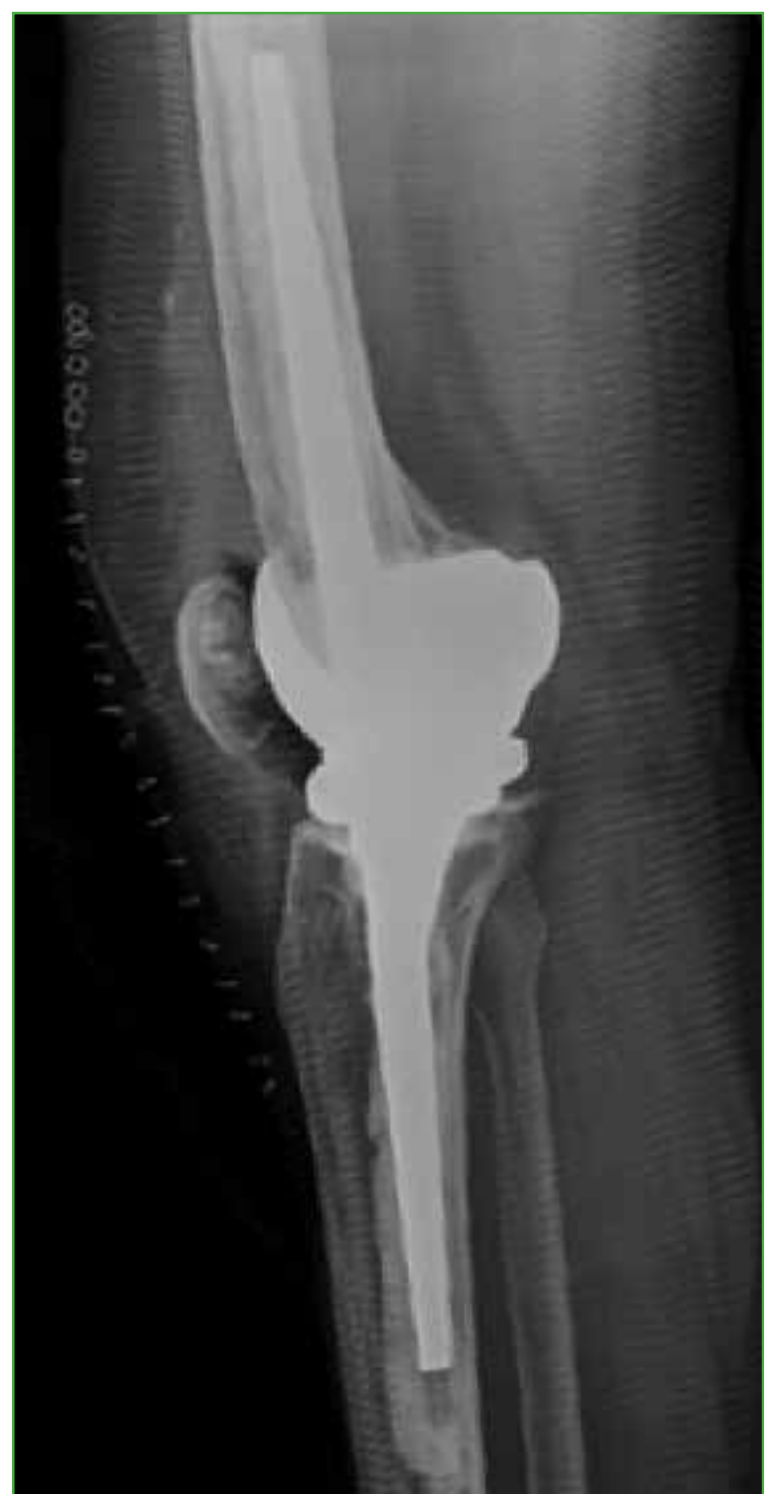

Figura 11. Radiografía de perfil de la revisión. Eje adecuado.

\section{DISCUSIÓN}

Estos dos casos tienen características en común; por un lado, la rotura del poste de polietileno, con el mismo modelo de prótesis estabilizada a posterior y, por el otro, la presentación clínica con recurvatum.

En el genu recurvatum no protésico, debemos distinguir entre pacientes con compromiso neuromuscular o sin él. Cuando hay compromiso, coexisten desequilibrios musculares y laxitud ligamentaria, con frecuencia, debilidad del cuádriceps, por lo cual el paciente lleva la rodilla a la hiperextensión para poder caminar generando progresivamente un recurvatum..$^{2,5,6}$ 
Si no hay enfermedad neurológica, el recurvatum puede asociarse a valgos severos y también a laxitud ligamentaria propia de la artritis reumatoide. ${ }^{5}$

El primer paciente tenía debilidad de cuádriceps y la segunda, una importante laxitud ligamentaria asociada a la artritis reumatoide. En ambos, la marcha era con hiperextensión.

En estos casos, el mecanismo de falla de la fractura del poste de polietileno se originó por el impacto anterior del poste con el componente femoral, con un desgaste excesivo sobre la base de su cara anterior. ${ }^{7,8}$

En 2009, Lim y cols. comunicaron un caso similar a los nuestros: una mujer de 72 años con antecedente de accidente cerebrovascular y debilidad muscular que fue sometida a una artroplastia de rodilla con prótesis Scorpio®; a los 14 meses, se produjo la rotura del poste y la presentación clínica con recurvatum. Los autores cambiaron el polietileno por uno de mayor grosor $\mathrm{y}$, a los tres meses, durante una artroscopia para la resección de un fragmento de rótula, observaron que la cara anterior del poste ya presentaba demarcación. ${ }^{9}$

En 2010, Lachiewicz publicó una revisión bibliográfica y halló 27 casos de esta complicación en 18 artículos, entre 2000 y 2010. Los pacientes acudieron con inestabilidad y dolor, no hace referencia al recurvatum, que fue la forma de presentación en nuestros casos. ${ }^{10} \mathrm{Si}$ bien nuestros pacientes tenían el mismo modelo de prótesis, en este grupo de 27 roturas de postes tibiales, hubo ocho diseños diferentes de cinco fabricantes distintos, lo que indica que esta complicación (la fractura del poste tibial) puede ocurrir con diferentes modelos de prótesis. ${ }^{11}$

Respecto del inserto tibial y su fabricación, nuestros pacientes tenían un inserto tibial de alto entrecruzamiento, pero en la escasa bibliografía al respecto, podemos encontrar que esto también puede ocurrir con los insertos de alto peso molecular.

En 2018, Diamond y cols. publican cinco casos de fractura del poste tibial en prótesis estabilizadas a posterior con polietileno de alto entrecruzamiento, y aconsejan no utilizar este tipo de polietileno. ${ }^{12}$

En 2017, el registro australiano informó que se observaron diferencias en la supervivencia de los polietilenos (alto peso molecular vs. alto entrecruzamiento) en prótesis con conservación de cruzados a favor de los segundos, pero no hallaron diferencias significativas en los modelos estabilizados a posterior (4,6, IC95\% 4,0-5,2 vs. 5,1, IC95\% 4,7-5,6, respectivamente). ${ }^{13}$

Asimismo, en un estudio de cohorte retrospectivo, Paxton y cols. comunicaron que la incidencia de revisión a los cinco años para la prótesis de rodilla estabilizada a posterior NexGen® (Zimmer® Inc., Warsaw, IN, EE.UU.), comparando el polietileno de alto entrecruzamiento y el polietileno de peso molecular ultra-alto, fue del 3,5\% y $2,5 \%$, respectivamente, sin diferencias significativas, mientras que no hubo revisiones por fracturas o fallas del inserto tibial. ${ }^{14}$

En los casos presentados, se llegó al diagnóstico mediante radiografías y un exhaustivo examen físico. Se ha comunicado el uso de la resonancia magnética o la artroscopia como herramientas diagnósticas en pacientes con inestabilidad en extensión sin recurvatum. ${ }^{9}$

Como tratamiento de esta complicación, se ha descrito la revisión con intercambio del inserto tibial, aumentando su grosor. Si bien es una opción válida, no tiene sustento en un seguimiento a largo plazo. ${ }^{9,10}$ Cuando la presentación clínica y la causa del fallo es el recurvatum, el aumento del grosor del polietileno puede causar una recidiva de la deformidad, como ocurrió en el caso publicado por Erceg y Raki. ${ }^{15}$

\section{CONCLUSIONES}

La rotura del poste del inserto tibial es una complicación poco común que puede ocurrir con prótesis de diferentes marcas, como se ha publicado. Ante la presentación clínica con inestabilidad en recurvatum y déficit muscular o laxitud ligamentaria, como en los dos casos presentados, la revisión con una prótesis abisagrada rotatoria logró buenos resultados.

Conflicto de intereses: Los autores no declaran conflictos de intereses.

ORCID de F. H. Lopreite: https://orcid.org/0000-0002-2065-8649 ORCID de G. Garabano: https://orcid.org/0000-0001-5936-0607 ORCID de F. Tillet: https://orcid.org/0000-0003-2658-9856
ORCID de J. Rodríguez: https://orcid.org/0000-0002-1089-3071 ORCID de H. del Sel: https://orcid.org/0000-0002-3655-1408 


\section{BIBLIOGRAFÍA}

1. Fehring TK, Odum S, Griffin WL, Bohannon Mason J, Nadaud M. Early failures in total knee arthroplasty. Clin Orthop Relat Res 2001;392:315-8. https://doi.org/10.1097/00003086-200111000-00041

2. Sharkey PF, Hozack WJ, Rothman RH, Shastri S, Jacoby SM. Why are total knee arthroplasties failing today? Clin Orthop Relat Res 2002;404:7-13. https://doi.org/10.1097/00003086-200211000-00003

3. Valle CJD, Della Valle CJ. Update from the American Association of Hip and Knee Surgeons 2012 Annual Meeting. J Bone Joint Surg Orthopaedic Highlights: Knee Surgery 2013; p.e2. https://doi.org/10.2106/jbjs.ks.1.00519

4. Abdel MP, Haas SB. The unstable knee: wobble and buckle. Bone Joint J Br 2014;96:112-4. https://doi.org/10.1302/0301-620X.96B11.34325

5. Pesciallo CA, Garabano G, Viollaz G, Oviedo A, Simesen H, del Sel H. Artroplastia total de rodilla en genu recurvatum. Rev Asoc Argent Ortop Traumatol 2011;76(3):250-3. http://www.aaot.org.ar/revista/2011/n3/Rev_Asoc_Argent_Ortop_Traumatol_2011_76(3)_250.pdf

6. Vedoya S, Garabano G, del Sel Hernán. Indicación y resultados de la prótesis con estabilidad aumentada en la artroplastia primaria de rodilla. Rev Asoc Argent Ortop Traumatol 2018;83(2):94-100. https://doi.org/10.15417/issn.1852-7434.2018.83.2.775

7. Hsu Y, Lin Ch-H, Shu GHF, Hsieh T-J, Huang Chen CK. Fracture of the polyethylene tibial post in the posteriorstabilized total knee prosthesis: arthrographic and CT arthrographic diagnosis. Skeletal Radiol 2019;48(7):1145-8. https://doi.org/10.1007/s00256-019-03173-5

8. Li G, Papannagari R, Most E, Park SE, Johnson T, Tanamal L, Rubash HE. Anterior tibial post impingment in a posterior stabilized total knee arthroplasty. J Orthop Res 2005;23(3):536-41. https://doi.org/10.1016/j.orthres.2004.09.005

9. Lim HC, Bae JH, Hwang JH, Kim SJ, Yoon JY. Fracture of a polyethylene tibial post in a Scorpio posteriorstabilized knee prosthesis. Clin Orthop Surg 2009;1:118-21. https://doi.org/10.4055/cios.2009.1.2.118

10. Lachiewicz PF. How to treat a tibial post fracture in total knee arthroplasty? Clin Orthop Relat Res 2011;469:170915. https://doi.org/10.1007/s11999-010-1609-9

11. Furman BD, Lipman J, Kligman M, Wright TM, Haas SB. Tibial post wear in posterior-stabilized knee replacements is design-dependent. Clin Orthop Relat Res 2008;466:2650-5. https://doi.org/10.1007/s11999-008-0422-1

12. Diamond OJ, Howard L, Masri B. Five cases of tibial post fracture in posterior stabilized total knee arthroplasty using Prolong highly cross-linked polyethylene. Knee 2018;25:657-62. htpps://doi.org/10.1016/j.knee.2018.05.005

13. Annual Report 2017. Australian National Joint Registry Annual Report. Disponible en: https://aoanjrr.sahmri.com/en/annual-reports-2017

14. Paxton EW, Inacio MCS, Kurtz S, Love R, Cafri G, Namba RS. Is there a difference in total knee arthroplasty risk of revision in highly cross-linked versus conventional polyethylene? Clin Orthop Relat Res 2015;473:999-1008. https://doi/org/10.1007/s11999-014-4046-3

15. Erceg M, Raki M. Genu recurvatum as a complication after total knee arthroplasty. Acta Clin Croat 2012;51:265-8. PMID: 23115953 